\title{
Effect of Gum-Chewing after Cesarean Section on Gastrointestinal Function Recovery: A Systematic Review and Meta-Analysis of Randomized Trials
}

\author{
Liu Zhang, Yanlin Heng*, Hong Hu, Xin Liang \\ Department of Medicine, Yangtze University, Jingzhou, China \\ Email: ^575966059@qq.com
}

How to cite this paper: Zhang, L., Heng, Y.L., Hu, H. and Liang, X. (2019) Effect of Gum-Chewing after Cesarean Section on Gastrointestinal Function Recovery: A Systematic Review and Meta-Analysis of Randomized Trials. Yangtze Medicine, 3, 79-89.

https://doi.org/10.4236/ym.2019.32009

Received: October 21, 2018

Accepted: March 19, 2019

Published: March 22, 2019

Copyright $\odot 2019$ by author(s) and Scientific Research Publishing Inc. This work is licensed under the Creative Commons Attribution International License (CC BY 4.0).

http://creativecommons.org/licenses/by/4.0/

\begin{abstract}
Objective: To evaluate the effect of chewing sugar-free gum on gastrointestinal function recovery after cesarean section. Methods: Randomized controlled trials comparing the use of chewing gum in postoperative recovery with a control group were retrieved from the databases including Pubmed, Cochrane Library, Science Direct, Web of Science, CBM, CNKI, Wanfang and VIP, et al. After screening literatures, evaluating the quality of studies, extracting data, the RevMan5.3 software was used for data analysis. Results: A total of 13 RCTs including 2233 patients were analyzed in the study. The results showed that chewing gum after cesarean delivery can effectively shorten the recovery time of bowel sounds, passage of flatus and first defecation (all $P<0.05$ ). Conclusion: Chewing sugar-free gum after cesarean section can promote the early recovery of gastrointestinal function, but the side effects of chewing gum are still unclear, which needs more clinical, large sample and high-quality studies to further verify.
\end{abstract}

\section{Keywords}

Cesarean Section, Gum-Chewing, Gastrointestinal Function, Randomized Controlled Trial, Systematic Review

\section{Introduction}

In recent years, with the development of medical technology and related support policies, the cesarean section rate in the world has tended to rise [1]. According to the survey conducted by WHO in 2010, the rate of cesarean section in China 
is the highest among 9 countries in Asia, up to 46\%. From 2008 to 2014, the total annual delivery rate of cesarean section in China increased by $34.9 \%$ [2]. However, cesarean section is prone to cause a series of postoperative complications: Postoperative ileus is an impaired condition of gastrointestinal, most likely to occur with day 4 postoperatively; the average incidence of postoperative ileus is about $20 \%$ [3]. A large number of studies have shown that early feeding can effectively promote the recovery of gastrointestinal function after cesarean section, but some patients performed high intolerance [4] [5], and delayed feeding is related to increase cell break-down, delay the time of wound healing and increase the risk of infection, thereby increasing medical costs [6] [7]. As early as 1992, Soffer pointed out that sham feeding could promote the secretion of saliva and pancreatic juice through the head-vagus reflex, thus promoting the early recovery of gastrointestinal function [8]. More studies have shown that chewing gum after abdominal surgery, including cesarean section, can effectively promote early recovery of gastrointestinal function and reduce postoperative complications [9]-[23]. At present, domestic meta-analysis is required more trials to provide sufficient evidence. The aim of this study was to evaluate the effect of chewing gum on the early recovery of gastrointestinal function after cesarean section by the method of evidence-based medicine, and provide a basis for further clinical exploration of the early recovery of gastrointestinal function in patients after cesarean section.

\section{Materials and Methods}

\subsection{Inclusion Criteria}

1) Study Design: randomized controlled trials (RCTs) about chewing gum in early stage after cesarean section were included in Chinese and English. 2) Participants: after cesarean section, the patients were in good health, conscious and had no serious complications (such as gestational hypertension, severe eclampsia, diabetes mellitus, etc.). 3) Interventions: the intervention group chewed sugar free gum after cesarean section, while the control group received routine nursing. 4) Outcome indicators (satisfying one of them): a) recovery time of bowel sounds; b) first time of passage of flatus; c) first time of defecation; d) first feeling of hunger.

\subsection{Exclusion Criteria}

The studies in which has one of the following conditions will be excluded, the interventions consisted of chewing gum in combination with other interventions; have no access to the original literature; incorrect use of data collection and statistical methods.

\subsection{Search Strategy}

We tried to plan, perform and report this meta-analysis in comply with PRISMA guideline [24]. Foreign language databases and Chinese databases were searched 
from their inception until July 2018 for randomized controlled trials about chewing gum in early stage after cesarean section, included Pubmed, Cochrane Library, Web of Science, Science Direct, Chinese Biomedical Literature Database (CBM), China National Knowledge Infrastructure(CNKI), WanFang database and VIP database, etc. Searchterms: "cesarean section/delivery", "gum-chewing/chewing", "randomized controlled trial/RCT" and "gastrointestinal function/gastrointestinal complications", and Mesh keywords, free words and Boolean logic operator conjunctions are combined in accordance to the instructions of all kinds of database. Retrieval words of similar meaning, such as "cesarean section" and "cesarean delivery" are linked with "or", but "cesarean section" and "gum-chewing" are linked with "and". In addition, the references of the retrieval studies and previous reviews were reviewed, computer retrieval is the main method, supplemented by the secondary literature traceability method to expand the retrieval, so as to avoid missing the studies not captured by electronic searches.

\subsection{Study Selection and Quality Evaluation}

1) Study screening and data extraction: According to the consistent inclusion and exclusion criteria, the data was selected and extracted by two researchers independently, the two researchers have learned knowledge about evidence-based medicine, and received standardized training. Extract contents including basic information (author, year of publication, country, baseline situation, etc.), research design, sample size, interventions, outcome indicators, etc. Any disagreement was resolved by discussion with a third reviewer. 2) Quality assessment: the two researchers independently evaluated the included studies according to Cochrane Assessment Manual 5.1.0 Assessment Criteria. The evaluation items included: random sequence generation, allocation hiding, blind method, data integrity of outcome indicators, selective reporting of findings and other sources of bias. A: fully meet the above criteria, the risk of a variety of bias is low; B: partially meet the above criteria, bias risk is moderate; $\mathrm{C}$ : completely dissatisfied with the above criteria, bias risk is higher. Only " $\mathrm{A}$ " and " $\mathrm{B}$ " studies were included in this study.

\subsection{Statistical Analysis}

Meta-analysis was performed using the RevMan5.3 software, provided by Cochrane collaboration network. Weighted mean difference (MD) was used for measurement data, such as recovery time of bowel sounds, first time of passage of flatus, first time of defecation, first feeling of hunger. Relative Risk (RR) was used for enumeration data. 95\% CI was calculated for all analyses. Firstly, Chi-square $\left(X^{2}\right)$ was used to confirm whether there was heterogeneity among the studies. If there was no significant statistical heterogeneity $(P>0.1, P<50 \%)$, the fixed effect model was adopted. If there was heterogeneity $(P<0.1, P \geq$ $50 \%$ ), the random effect model was used to combine with the effect size. De- 
scriptive analysis was adopted if heterogeneity was too obvious and can't be judged.

\section{Results}

\subsection{Study Selection and Study Characteristics}

A total of 443 trials about chewing-gum after cesarean section were initially retrieved, and 49 papers were included through preliminary reading literature and abstracts, removing summaries, case reports and small methods. Finally, 13 trials were assessed for eligibility after further reading. The flow of study identification is shown in Figure 1. Table 1 shows the characteristics of the included studies. The intervention group chewed sugar-free gum after cesarean section, while the control group received routine nursing, i.e., fluidized diet after a wake anesthesia or the first exhaust after cesarean section and gradually transited to general diet.

Table 1. Characteristics of the included studies.

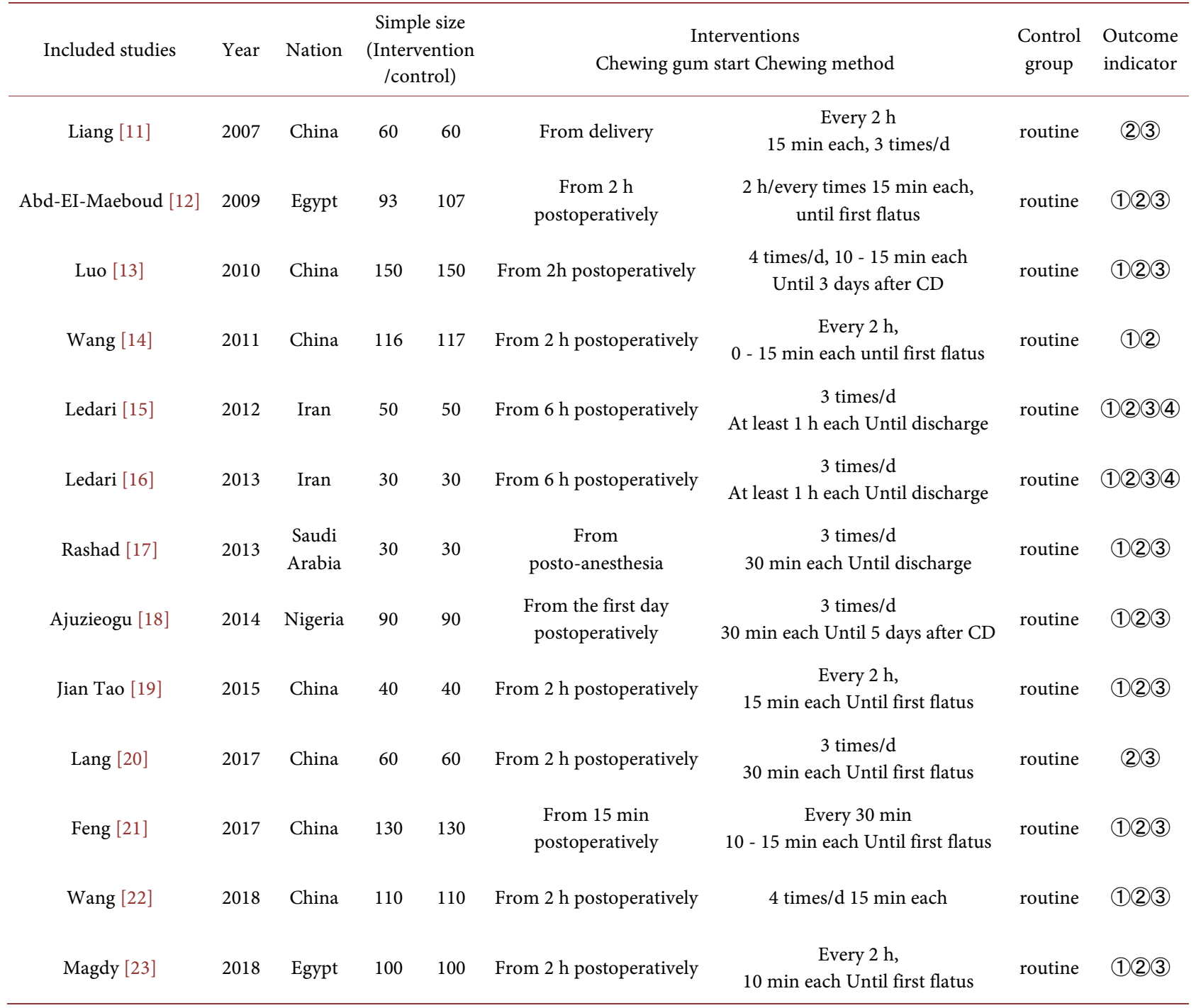

(1): recovery time of bowel sounds; (2): the time of first passage of flatus; (3): the time of first defection; (4): the time of first feeling of hunger. 


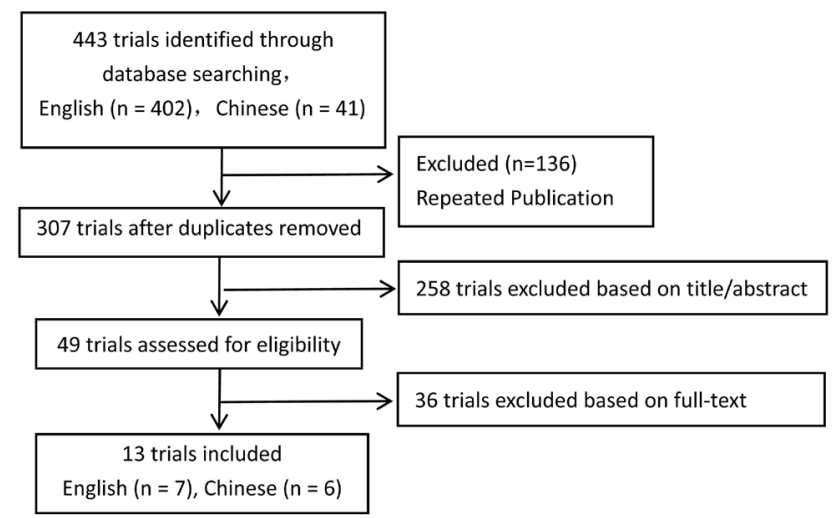

Figure 1. Flow diagram of studies identified in the systematic review.

\subsection{Methodological Quality Evaluation of the Literature}

Therefore 13 trials, including 2233 patients were analyzed, of which 7 trials were in English and 6 in Chinese. The methodological quality of the study was evaluated in Table 2. It showed the qualities of the 3 studies were grade A and 10 were grade $\mathrm{B}$.

\subsection{Results of Systematic Reviews}

\subsubsection{Effect of Chewing Sugar-Free Gum on Bowel Sounds}

11 studies [10]-[17] [19] [20] [21] reported the effect of chewing gum post operatively on the recovery time of bowel sounds. 6 trials [10] [11] [12] [17] [20] [21] chewing gum within $2 \mathrm{~h}$ of delivery, and were analyzed quantitatively, the combined results showed that there was heterogeneity between the studies $(P<$ $0.00001, I^{2}=97 \%$ ), so a randomized effect model was used for meta-analysis. The results showed that the recovery time of bowel sounds of postoperatively chewing gum was earlier than that of routine nursing group, and the difference was statistically significant $[M D=-6.27,95 \% C I(-7.98,-4.56), Z=7.18, P<$ 0.00001 ], as shown in Figure 2. The sensitivity analysis showed the difference in each group had statistically significant after removing the study with the smallest sample size, indicating that the results were stable. And Ledari et al. [13] [14] [15] [16] [19] reported that chewing gum at different times after surgery the results also showed statistically significant.

\subsubsection{Effect of Chewing Sugar-Free Gum on the Passage of Flatus}

The effect of postoperatively chewing gum on anal exhaust time was reported in 13 trials, 7 studies [10] [11] [12] [17] [18] [20] [21] could be quantitatively analyzed. Heterogeneity was examined by Chi-square, found there was heterogeneity in each group $\left(P<0.00001, P^{2}=99 \%\right)$, so the random effect model was adopted. The results showed that the first time of passage of flatus in chewing gum group was shorter than that in control group, and the difference was statistically significant $[M D=-8.66,95 \% C I(-14.12,-3.20), Z=3.11, P=0.002]$, as shown in Figure 3. At the same time, in Liang Junhua and other studies [9] [13] [14] [15] [16] [19], the results were different between the two groups $(P<0.05)$. 


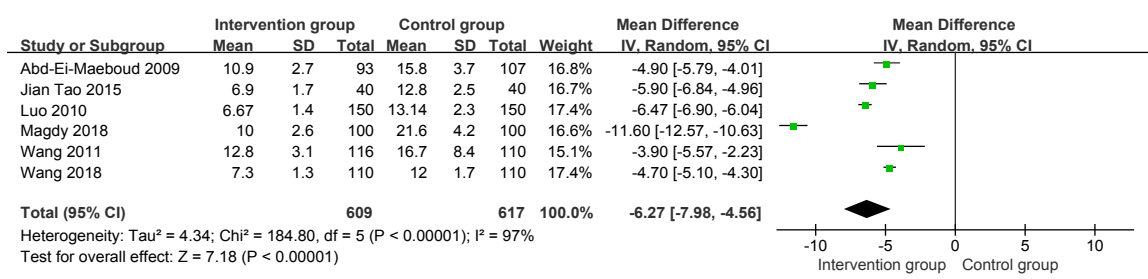

Figure 2. Effect of chewing sugar-free gum on bowel sounds.

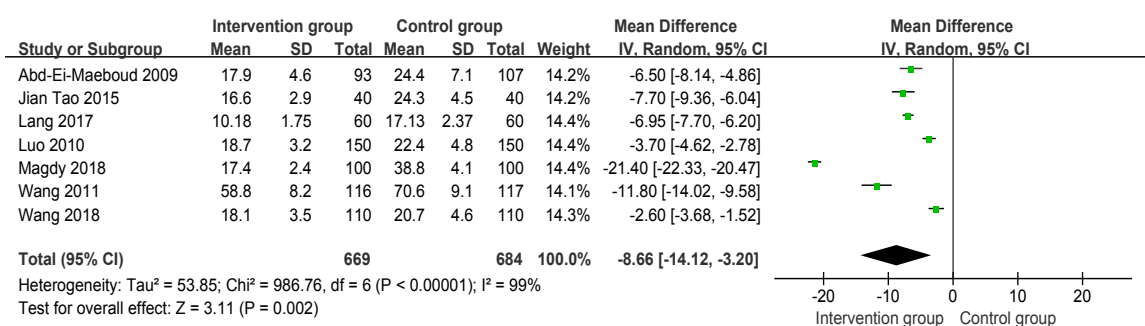

Figure 3. Effect of chewing sugar-free gum on the passage of flatus.

Table 2. Assessment of risk of bias.

\begin{tabular}{ccccccccc}
\hline Inclusion studies & $\begin{array}{c}\text { Random } \\
\text { sequence } \\
\text { generation }\end{array}$ & Blinding of & $\begin{array}{c}\text { Blinding of } \\
\text { participants } \\
\text { outcome } \\
\text { assessment }\end{array}$ & $\begin{array}{c}\text { outcome } \\
\text { data }\end{array}$ & $\begin{array}{c}\text { Selective } \\
\text { reporting }\end{array}$ & $\begin{array}{c}\text { Other } \\
\text { bias }\end{array}$ & $\begin{array}{c}\text { Quality } \\
\text { grade }\end{array}$ \\
\hline Liang [11] & unclear & unclear & unclear & low & low & unclear & B \\
Abd-EI-Maeboud [12] & low & low & low & low & low & low & A \\
Luo [13] & unclear & unclear & unclear & low & low & unclear & B \\
Wang [14] & low & unclear & unclear & low & low & low & B \\
Ledari [15] & low & unclear & low & low & low & low & B \\
Ledari [16] & unclear & unclear & unclear & low & low & unclear & B \\
Rashad [17] & unclear & unclear & unclear & low & low & unclear & B \\
Ajuzieogu [18] & low & low & low & low & low & low & A \\
Jian Tao [19] & low & low & low & low & low & low & A \\
Lang [20] & low & unclear & unclear & low & low & low & B \\
Feng [21] & low & unclear & unclear & low & low & low & B \\
Wang [22] & low & unclear & unclear & low & low & low & B \\
Magdy [23] & low & unclear & unclear & low & low & low & B
\end{tabular}

A: Fully meet the above criteria, the risk of a variety of bias is low; B: Partially meet the above criteria, bias risk is moderate.

\subsubsection{Effect of Chewing Gum on the Time of First Defecation}

12 studies [9] [10] [11] [13]-[21] reported the effect of postoperatively chewing gum on the first time of defecation. 6 studies [10] [11] [17] [18] [20] [21] were analyzed quantitatively. Heterogeneity showed that random effect model should be selected to analyze $(P<0.00001, P=99 \%)$. The results showed that the defecation time of the chewing sugar-free gum group was earlier than that of the routine nursing group, and the difference was statistically significant $[M D=$ 
$-9.06,95 \% C I(-15.59,-2.53), Z=2.72, P=0.007]$, as shown in Figure 4 . In the meanwhile, in the studies of Liang Junhua et al. [9] [13] [14] [15] [16] [19], the difference was also statistically significant, indicating that early chewing gum can promote defecation time ahead.

\subsubsection{Effect of Chewing Gum on the Time of First Feeling of Hunger}

Two trials [13] [14] reported the effect of postoperatively chewing gum on the time of first feeling of hunger. There was no heterogeneity between two studies ( $\left.P=0.18, P^{2}=43 \%\right)$, therefore, the fixed effect model was used. The results showed that chewing gum after cesarean section can promote patients feel hunger and then start early feeding $[M D=-3.75,95 \% C I(-5.99,-1.50), Z=3.27, P$ $=0.001]$, as shown in Figure 5 .

\section{Discussion}

\subsection{Methodological Quality of the Included Studies}

13 trials were included in this study, the quality of the included trials was medium. 9 trials [12] [14] [15] [18]-[23] produced random sequences by computer-ganerated random sequence. 4 trials [11] [13] [16] [17] just mentioned random allocation, how random sequence generated was unclear, because it was difficult to achieve double-blind for intervention group and control group, three studies [12] [18] [19] conducted a single-blind. One trial [15] reported the missing rate and missing reasons. The baseline datas of age, gestational age, education level and parity were compared between the intervention group and the control group. The results showed that the baseline datas were comparable between the two groups $(P>0.05)$.

However, the clinical heterogeneity between these studies was large, and the specific program of chewing gum was obviously different. Most studies began chewing gum within $2 \mathrm{~h}$ of cesarean delivery, while a few studies [15] [16] [17] [18] began after $6 \mathrm{~h}$ or more of surgery, whether it made sense in early stage was unclear. Xie Minyi et al., [25] investigated the effect of chewing gum at different

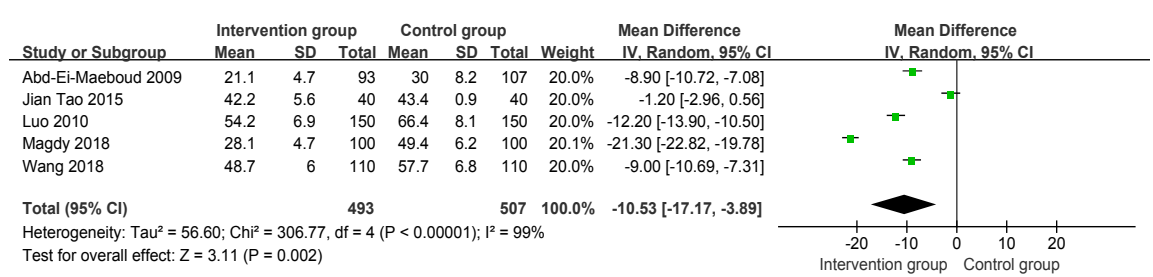

Figure 4. Effect of chewing gum on the time of first defecation.

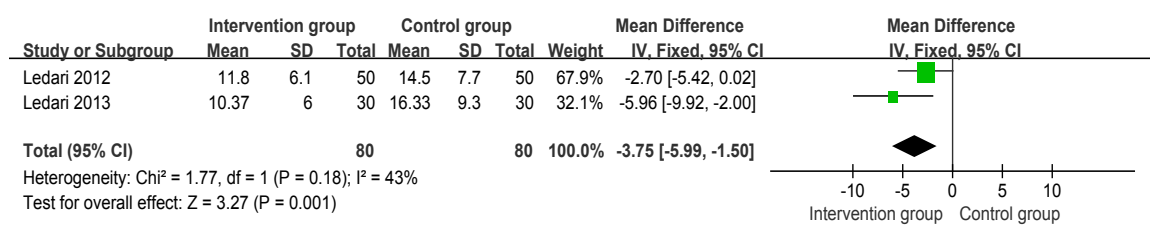

Figure 5. Effect of chewing gum on the time of first feeling of hunger. 
time windows on the recovery of gastrointestinal function after gastrointestinal surgery, the results showed that the level of serum gastrin in group A (chewing gum at postoperative $2 \mathrm{~h}$ ) were higher than those in group B (chewing gum at postoperative $6 \mathrm{~h}$ ) and the incidence of abdominal distention was lower in group A than that in group B $(P<0.05)$, indicated that chewing gum the earlier, the effect is better. Some studies [15] [16] [17] [18] stopped chewing gum after discharge or 5 days of delivery, which was different from the general study, and it was not clear whether chewing gum was still meaningful after patients returned to normal diet, while in some studies [15] [16], patients chewed gum for more than one hour, the time of chewing was too long and the frequency was too much can easily lead to masseter muscle fatigue, abdominal distension, dizziness, nausea and other adverse reaction.

\subsection{Effect of Chewing Gum on Gastrointestinal Function Recovery after Cesarean Section}

The gastrointestinal adverse reactions such as nausea, vomiting and abdominal distention are more likely to occur during the period from inhibition to full recovery of gastrointestinal function after cesarean section. Recovering gastrointestinal function as soon as possible is of great significance in preventing gastrointestinal complications after cesarean section and promoting the health of maternal and infant. Chewing gum, as a non pharmacological methods is convenient and safe, it not only doesn't increase the burden of gastrointestinal tract and cause a series of complications, but also stimulate gastrointestinal motility, which is easily accepted by patients. Studies [14] [26] have showed that chewing gum can significantly increase the levels of gastrin and motilin in peripheral gastrointestinal hormones on the first and second day after operation, thereby promoting gastrointestinal motility. And, the majority of cesarean section objects are young mothers with good digestive function, the way of anesthesia is epidural anesthesia, postoperative patients have clear consciousness, and the possibility of vomiting, asphyxia and other complications caused by eating are low. It indicates that maternal have the physiological basis of early feeding, it's safe enough for maternal to chewing gum after delivery [27].

Related meta-analysis [28] [29] showed that chewing gum could promote the early recovery of gastrointestinal function after cesarean section, but the start time and frequency of chewing gum were different. In most of the included studies, chewing gum was given within $2 \mathrm{~h}$ of delivery, at least 3 times one day, 15 30 minutes each time, and until the first bowel sounds was heard. Quantitative analysis of the combined studies was carried out. Otherwise, descriptive analysis was selected. The results showed that chewing gum could promote the early recovery of maternal gastrointestinal function. It was consistent with the results of review by Perriera et al. [30]. And, Liang Junhua et al., [11] [21] [22] showed that chewing gum postoperative can effectively reduce the incidence of nausea, vomiting, abdominal distention and other adverse gastrointestinal reactions. Abd-EI-Maeboud et al., [12] [20] [21] showed that chewing gum after surgery 
can shorten hospitalization time. However, study [18] showed that it has no significant difference in hospitalization time compared with control group. Study [31] showed that mastication activates histamine nervous system conduction signals and promotes histamine production, thereby reducing hunger by inhibiting food intake by receptors located in the center of satiety. Whether the feeling of hunger can be used as a criterion for evaluating gastrointestinal functional recovery needs to be further discussed.

\subsection{Limitations of This Systematic Review}

Because this study only retrieves the published Chinese and English trials, some trials may not be included. There are great differences in the sample and methodology of the included studies, and the intervention schemes are not consistent, which may have a certain impact on the results of the study. Sugar-free gums containing artificial sweetener sorbitol and other alcohols may have side effects such as bloating, flatulence and abdominal colic [32]. However, in the 13 trials, there was no study reported the side effect of chewing gum, the effect of chewing gum on the recovery of gastrointestinal function remains to be further explored.

\section{Conclusion}

Chewing gum as a simple and convenient intervention can effectively promote the recovery of bowel sounds after cesarean section, and shorten the first time of passage of flatus and the defecation. In the future, we can expand the sample size and carry out high-quality randomized controlled trials to validate the effect of standardized chewing gum intervention program (start time, frequency, chewing dose, duration, etc.) on promoting the recovery of maternal gastrointestinal function.

\section{Conflicts of Interest}

The authors declare no conflicts of interest regarding the publication of this paper.

\section{References}

[1] Degani, N. and Sikich, N. (2015) Caesarean Delivery Rate Review: An Evidence-Based Analysis. Ontario Health Technology Assessment Series, 15, 1-58.

[2] Li, H.T., Luo, S., Trasande, L., et al. (2017) Geographic Variations and Temporal Trends in Cesarean Delivery Rates in China, 2008-2014. JAMA, 317, 69-76. https://doi.org/10.1001/jama.2016.18663

[3] Vather, R., Trivedi, S. and Bissett, I. (2013) Defining Postoperative Ileus: Results of a Systematic Review and Global Survey. Journal of Gastrointestinal Surgery, 17, 962-972. https://doi.org/10.1007/s11605-013-2148-y

[4] Kathpalia, S.K. (2017) Early Maternal Feeding versus Traditional Delayed Feeding after Cesarean Section: A Pilot Study. Journal of Obstetrics and Gynecology of India, 67, 178-182. https://doi.org/10.1007/s13224-016-0949-0 
[5] Huang, H., Wang, H. and He, M. (2016) Early Oral Feeding Compared with Delayed Oral Feeding after Cesarean Section: A Meta-Analysis. Journal of Maternal-Fetal \& Neonatal Medicine, 29, 423-429. https://doi.org/10.3109/14767058.2014.1002765

[6] Dehcheshmeh, D.F., Salehian, T., Gangi, F. and Beigi, M. (2011) The Effect of Chewing Sugar Free Gum after Elective Cesarean-Delivery on Return of Bowel Function in Primiparous Women. QJRMS, 4, 16-20.

[7] Han-Geurts, I.J., Jeekel, J., Tilanus, H.W. and Brouwer, K.J. (2001) Randomized Clinical Trial of Patient-Controlled versus Fixed Regimen Feeding after Elective Abdominal Surgery. British Journal of Surgery, 88, 1578-1582. https://doi.org/10.1046/j.0007-1323.2001.01934.x

[8] Soffer, E.E and Adrian, T.E. (1992) Effect of Meal Composition and Sham Feeding on Duodenojejunal Motility in Humans. Digestive Diseases and Sciences, 37, 1009-1014. https://doi.org/10.1007/BF01300279

[9] Lambrichts, D.P.V., Boersema, G.S.A., et al. (2017) Nicotine Chewing Gum for the Prevention of Postoperative Ileus after Colorectal Surgery: A Multicenter, Double-Blind, Randomised, Controlled Pilot Study. International Journal of Colorectal Disease, 32, 1267-1275. https://doi.org/10.1007/s00384-017-2839-z

[10] Ge, B.J., Zhao, H., Lin, R., et al. (2017) Influence of Gum-Chewing on Postoperative Bowel Activity after Laparoscopic Surgery for Gastric Cancer a Randomized Controlled Trial. Medicine, 96, 1-5. https://doi.org/10.1097/MD.0000000000006501

[11] Liang, J.-H., Gao, T., Han, W.-W., et al. (2007) The Clinical Observation of Enhancing Recovery of Gastrointestinal. Journal of Tong Ji University (Medical Science), 28, 81-83.

[12] Abd-El-Maeboud, K.H., Ibrahim, M.I., et al. (2009) Gum Chewing Stimulates Early Return of Bowel Motility after Caesarean Section. BJOG, 116, 1334-1339. https://doi.org/10.1111/j.1471-0528.2009.02225.x

[13] Luo, S.-Q., Wu, C.-L., Yang, X.-M., et al. (2010) Effect of Chewing gum after Cesarean Section on Restoration of Gastrointestinal Function. Chinese Journal of Modern Nursing, 16, 2948-2949.

[14] Wang, X., Ren, Y., Qin, X.Y., et al. (2011) Influence of Sham Feeding on Motilin and Evacuating Time after Accepting Cesarean Section. Chinese Nursing Research, $25,682-683$.

[15] Ledari, F.M., Barat, S. and Delavar, M.A. (2012) Chewing Gums Has Stimulatory Effects on Bowel Function in Patients Undergoing Cesarean Section: A Randomized Controlled Trial. Bosnian Journal of Basic Medical Sciences, 12, 265-268. https://doi.org/10.17305/bjbms.2012.2452

[16] Ledari, F.M., Barat, S., Delavar, M.A., et al. (2013) Chewing Sugar-Free Gum Reduces Ileus after Cesarean Section Innulliparous Women: A Randomized Clinical Trial. Iranian Red Crescent Medical Journal, 15, 330-333. https://doi.org/10.5812/ircmj.6458

[17] Rashad, W., Suliman, A. and Yousef, A.L. (2013) Effect of Sugarlessgum Chewing on Intestinal Movement after Cesarean Section. Life Science Journal, 10, 3257-3261.

[18] Ajuzieogu, O.V., Amucheazi, A., Ezike, H.A., et al. (2014) The Efficacy of Chewing Gum on Postoperative Ileus Following Cesarean Section in Enugu, South East Nigeria: A Randomized Controlled Clinical Trial. Nigerian Journal of Clinical Practice, 17, 739-742. https://doi.org/10.4103/1119-3077.144388

[19] Lee, J.T., Lin, J.-R., et al. (2015) The Role of Xylitol Gum Chewing in Restoring Postoperative Bowel Activity after Cesarean Section. Biological Research for Nurs- 
ing, 1-6.

[20] Lang, S. and Chen, X. (2017) Influence of Chewing-Gum after Cesarean Section on Restoration of Gastrointestinal Function. Psychological Doctor, 23, 337-338.

[21] Feng, H. (2017) Effect of Chewing Gum after on Gastrointestinal Function Recovery of Cesarean Section. Contemporary Medical Symposium, 15, 152-153.

[22] Wang, P. (2018) Influence of Chewing Gum after Cesarean Section on Restoration of Gastrointestinal Function. Chinese Journal of Rural Medicine and Pharmacy, 25, 7-8.

[23] Magdy, R.A., Waleed Ali, S.A., Rasha, E.K., et al. (2018) Efficacy of Three Different Regimens in Recovery of Bowel Function Following Elective Cesarean Section: A Randomized Trial. Journal of Perinatal Medicine, 46, 786-790.

[24] Liberati, A., Altman, D.G., Tetzlaff, J., et al. (2009) The PRISMA Statement for Reporting Systematic Reviews and Meta-Analyses of Studies That Evaluate Health Care Interventions: Explanation and Elaboration. PLOS Medicine, 6, 1-27. https://doi.org/10.1371/journal.pmed.1000100

[25] Xie, M., Ke, Y., Xie, R., et al. (2015) Study of Chewing Gum on Gastrointestinal Recovery after Gastrointestinal Surgery at Different Therapy Time Window. Chinese Nursing Management, 15, 659-660.

[26] Ren, Y., Qin, X., Wang, X., et al. (2011) Influence of Sham Feeding on Gastrin of Puerpera after Undergoing Cesarean Section. Chinese Nursing Research, 25, 1923-1924.

[27] Jeffrey, A. and Clark, V. (2011) The Anaesthetic Management of Caesarean Section in the Interventional Radiology Suite. Current Opinion in Anaesthesiology, 24, 439-444. https://doi.org/10.1097/ACO.0b013e32834811d4

[28] Ciardulli, A., Saccone, G., et al. (2017) Chewing Gumimproves Postoperative Recovery of Gastrointestinal Function after Cesarean Delivery: A Systematic Review and Meta-Analysis of Randomized Trials. The Journal of Maternal-Fetal \& Neonatal Medicine, 31, 1924-1932. https://doi.org/10.1080/14767058.2017.1330883

[29] Yuan, Y., Zhao, H., He, J., et al. (2011) Chewing Gum in Promoting Bowel Recovery after Cesarean Section: A Systematic Review. Chinese Journal of Evidence-Based Medicine, 11, 427-432.

[30] Perriera, G., Morais, E., Riera, R., Porfirio, G.J., et al. (2016) Chewing Gum for Enhancing Early Recovery of Bowel Function after Caesarean Section. Cochrane Database of Systematic Reviews, No. 10, CD011562.

[31] Tandeter, H. (2009) Hypothesis: Hexitols in Chewing Gum May Play a Role in Reducing Postoperative Ileus. Medical Hypotheses, 72, 39-40.

https://doi.org/10.1016/j.mehy.2008.06.044

[32] Zeng, H., Tan, Y. and Liu, Z. (2006) Research Progress on the Role and Mechanism of Histamine in the Pathogenesis of Obesity. Central South Pharmacy, 4, 218-221. 